\title{
ARTICLE
}

\section{Death by PowerPoint: alternatives for life}

\author{
Richard M. Duffy \& Marian Henry
}

Richard M. Duffy is a senior registrar in liaison psychiatry in Our Lady of Lourdes Hospital Drogheda and an occasional lecturer at the School of Medicine and Medical Science, University College Dublin. He was responsible for introducing the software Prezi into the teaching of psychiatry and carried out research into students' experience of this. Marian Henry is an occasional lecturer at St Patrick's College of Education, Dublin. Her doctoral thesis explored how learning is changing in a digital age. She designs and develops digital media literacy courses, and facilitates professional development for teachers.

Correspondence Dr Richard M. Duffy, Department of Adult Psychiatry, Mater Misericordiae University Hospital, 63 Eccles Stree Dublin 7, Ireland. Email: duffyrm@ gmail.com

\begin{abstract}
SUMMARY
The use of PowerPoint has become nearly ubiquitous in medical education and continuing professional development; however, many alternatives are emerging that can be used in its place. These may confer some advantages, but they also have potential drawbacks. It is helpful that educators are aware of these new presentation options and their pros and cons, including any financial implications and issues of data protection. This article considers the role of technology in teaching and learning, identifying underlying assumptions that are often made. It identifies and appraises technology that can be used with or instead of PowerPoint to best facilitate deep learning. The potential pedagogical benefits and practical limitations of these technologies are considered, and strategies are highlighted to maximise the impact of PowerPoint where it is the software of choice.
\end{abstract}

\section{LEARNING OBJECTIVES}

- Consider the role of information communication technology in teaching and learning

- Identify and appraise potential technology that may enhance the use of PowerPoint in educational settings

- Identify and appraise alternatives to standard presentation software that may aid the learning experience

\section{DECLARATION OF INTEREST}

None

Since its release by Microsoft in 1990, hundreds of millions of people have used PowerPoint to deliver information and convey ideas. In educational settings, PowerPoint is commonly used for largegroup teaching, but increasingly it is also being used in tutorials and small groups. Most of us have experienced the range of quality of these presentations - although some are engaging and thought-provoking, others leave the audience confused, overloaded or bored.

So much has been said about the overuse of PowerPoint that 'death by PowerPoint' is now a term in regular usage (Garber 2001); the Google Books Ngram Viewer (Michel 2011) shows a sharp increase in the use of the term since 1996. There has been much debate over whether PowerPoint is a powerful teaching tool or a hindrance to learning (Undrill 2013). Essentially, the discussion of PowerPoint and its effectiveness in teaching is indicative of a much broader discussion of how we use technology in learning environments. Even before the advent of PowerPoint, evidence existed questioning the effectiveness of lectures in facilitating deep learning (Dunkin 1983; Long 2014). In the 21st century, lecturers have access to a wide array of technological tools that can enhance the learning experience of their students. They are grappling with which technology is best to use, as well as when, why and how to use it. Although it is important to stay up to date with technological advances that could be used in medical education, it is also necessary to carefully evaluate the pros and cons of any such interventions (Hilty 2006) and reflect on their effectiveness and usefulness. This article seeks to address some of these themes that are prevalent for educators.

\section{Learning and technology}

The use of technology in learning environments is often presented as a fait accompli, the emphasis being on how we use technology as opposed to whether or why we should use it (Henry 2015). Historically, efforts to introduce technology into education can be described as being a 'solution in search of a problem' (Selwyn 2011: p. 57), whereby technology is implanted into education settings, with teachers expected to make use of it. According to this model, the technology comes first and a change in learning follows. However, there is little evidence to suggest that this is the case in practice. The literature on and research into educational technology is overpopulated with in-depth studies of small-scale, well-resourced projects that are as likely to indicate the 'Hawthorne effect' as to provide any direct evidence that educational technology is living up to the claims made on its behalf (Buckingham 2007). The Hawthorne effect refers to the sense that the novelty and enthusiasm associated with any innovation may be responsible for the perceived benefits it appears to bring about, as opposed to these benefits being 


\section{BOX 1 Information communication technologies for teaching and learning}

Coggle: https://coggle.it

FreeMind: https://sourceforge.net/projects/freemind

Google Slides: https://www.google.com/slides/about

Haiku Deck: https://www.haikudeck.com

Keynote: https://www.apple.com/mac/keynote

Popplet: www.popplet.com

PowToon: https://www.powtoon.com

Presentation Free 2013: www.ksosoft.com/product/

presentation-free.html

Prezi: www.prezi.com

RawShorts: www.rawshorts.com

directly caused by the technology. To say that information communication technology (ICT) changes or revolutionises learning is technological determinism - meaning that we see technology as causing change. ICT may play a part in change, but technologies cannot have an impact without people to use them and to appropriate them into their learning. As was found in a recent Nesta report, 'Technology has no impact on its own it all depends how we use it' (Stokes 2012: p. 8). Technology is part of the story, but in order for ICT to have a positive impact on learning, lecturers need to be informed users of it (Henry 2015). This means discerning the best use of a tool, or combination of tools, to inform, engage and motivate learners. The different tools described below all offer ways of combining images and words. What makes a teaching session successful depends more on the teacher and the students than on the software used. Despite this, we feel that there are distinct benefits to the media below, which are worth exploring and which may confer some pedagogical advantage to teachers and learners.

Various technologies have been used, with mixed effects (Clark 2011). Digital tools offer the opportunity to broaden and deepen the learning experience. Links to websites for all the ICT discussed below can be found in Box 1, and many of the pros and cons are summarised in Table 1. These tools allow for information to be presented in a number of different ways, thus catering for a variety of learning styles. The use of images, videos, mind maps, sound and interactive components can all improve engagement, recall and understanding (Clark 2011). Cantillon (2003) suggests that the medium should be tailored to the concepts and themes being taught. Some topics lend themselves to being depicted through mind maps, whereas some greatly benefit from the inclusion of videos. For example, the inclusion of videos in teaching about catatonia can help clarify the subject to students and make it more memorable. The presentation of the differential diagnosis of psychosis as a mind map can highlight to students how the different potential diagnoses are related; this can give them a visual tool to form differentials of their own. Cantillon (2003)

\section{TABLE 1 Advantages and disadvantages of alternatives to PowerPoint}

\begin{tabular}{|c|c|c|}
\hline Presentation software & Advantages & Disadvantages \\
\hline Apache OpenOffice & $\begin{array}{l}\text { Free } \\
\text { Downloadable } \\
\text { Highly compatible with PowerPoint }\end{array}$ & $\mathrm{n} / \mathrm{a}$ \\
\hline Google Slides & $\begin{array}{l}\text { Free } \\
\text { Cloud-based storage - great for sharing and remote editing } \\
\text { Highly compatible with PowerPoint } \\
\text { Can export as PDF }\end{array}$ & Cloud-based storage - may cause data protection problems \\
\hline Haiku Deck & $\begin{array}{l}\text { Free } \\
\text { Simple to use }\end{array}$ & Complicated to export files to PowerPoint \\
\hline Keynote & $\begin{array}{l}\text { Great video tutorials are available } \\
\text { Aesthetically superior to PowerPoint } \\
\text { Highly compatible with PowerPoint }\end{array}$ & Requires Apple (iOS) \\
\hline $\begin{array}{l}\text { Powtoon, RawShorts, Videoscribe and } \\
\text { other animation technologies }\end{array}$ & $\begin{array}{l}\text { Great for brief simple messages } \\
\text { Visual impact }\end{array}$ & $\begin{array}{l}\text { Complex to use } \\
\text { Extended features require subscription }\end{array}$ \\
\hline Presentation Free 2013 & $\begin{array}{l}\text { Free } \\
\text { Downloadable } \\
\text { Highly compatible with PowerPoint }\end{array}$ & $\mathrm{n} / \mathrm{a}$ \\
\hline Prezi & $\begin{array}{l}\text { Can provide a great overview } \\
\text { Puts information in context } \\
\text { Special package for students and educators } \\
\text { Cloud-based storage - great for sharing and remote editing }\end{array}$ & $\begin{array}{l}\text { More complex to use than PowerPoint } \\
\text { Cloud-based storage - may cause data protection problems } \\
\text { Cannot export files as PowerPoint }\end{array}$ \\
\hline
\end{tabular}


also suggests that the medium should encourage learning through interaction. Some options to foster interaction are discussed below.

There are three main challenges when using ICT. First, the technical skill and experience of the lecturer may be a limiting factor - any change to using a different medium or technology may require significant amounts of work in the early stages.

Second, the technical ability within the student body may vary - 'millennial' students approach learning differently from previous generations, being more comfortable with using technology (Beebe 2014); however, teachers must not assume that this is always the case. Active steps must be taken to induct students into use of the new technology, and information technology (IT) support should be made available to staff and students. Although research has been conducted in this area, it is still in its infancy and lacks clear guidelines.

Third, any new technology can sadly run into technical problems, often at the most inopportune times. This can often be the case with new software (Beebe 2014; Duffy 2014). It must be noted that there are serious limitations to presenting directly from files saved on the internet; even with a reliable connection, alerts, emails and software upgrades can interfere with a lecture. Whenever possible, the presentation should be saved as a local copy and should not be dependent on the internet.

\section{'Resuscitation' of PowerPoint}

The influence PowerPoint has had on education cannot and should not be underestimated. It has enabled us to convey information in new and varied ways. It can be used to clearly express ideas in a visual and engaging way. Presentations can be used as learning resources; hard copies of the slides are easy to create as handouts, and for many students they have partly replaced textbooks. Although people have been critical of PowerPoint, it is a powerful tool with huge potential. It is estimated that between 20 and 30 million PowerPoint presentations are given daily (Craig 2006). As it stands, everything else either builds on PowerPoint or is an alternative to it. In this section, we aim to cast light on ICT that can help realise the full potential of PowerPoint.

\section{Interactive technology}

Personal response systems (PRS) or electronic voting systems have been developed that allow students to answer questions and take polls en masse during a lecture. For example, a lecturer may pose the question: "What are the potential causes of cognitive decline?' Students can be presented with array of options and can vote from a range of answers. PRS have been incorporated into PowerPoint lectures (Revell 2010). It has been suggested that PRS facilitate active learning, foster participation, provide both students and lecturers with instant feedback and improve attendance (Duncan 2005; Revell 2010). It has been said that today's students may learn in different ways compared with previous generations (Beebe 2014) and consequently the provision of instant feedback with interactive technology is more important for 'millennials'. This argument, although it has merit, fails to recognise the potential strength of interactive technology. A PRS can allow the lecturer to get a sense of how well students understand what they need to - in real time, during the lecture, when they can actually do something about it. Gaining an understanding of students' ability and their current level of knowledge can greatly benefit a lecture and can help teachers avoid either cognitively overloading them (Sweller 1994) or covering information they already know. Students have expressed a strong desire to have interactive lectures even in large-group settings (Roopa 2013). It is important to consider the attention span and the baseline level of knowledge of the audience. Students' retention of knowledge in a lecture has been shown to reduce after 20 minutes (Bligh 2000; Long 2014). The inclusion of interactive components in presentations improves attention and can increase overall learning (Wessels 2007). Encouraging student participation can be difficult and intimidating for both teachers and students in larger groups. A PRS may be an easier way of facilitating this.

One of the leading current providers of electronic voting systems is TurningPoint. It was initially developed to work with a small hand-held response device. However, with the ubiquitous nature of smartphones, ResponseWare has been developed to link the TurningPoint software with smartphones rather than the hand-held 'clickers'. Many universities, including Bath, Ulster and Leicester, are currently using this software.

\section{Animation technology}

Technological advances and modern applications have made animation tools usable by and available to a wider audience. This type of software allows teachers to generate brief animations, not just movement during transition between slides. Although including animation can be more expensive and time-consuming, spectacular presentations can be produced. We have included animation software in this section on enhancing 
PowerPoint, as animations can be linked to or embedded in a presentation, but they can equally stand alone. Practically speaking, animations are more useful in replacing short 3-5 min presentations - for example, as an introduction to a conference, for an online video for students, or in providing patient information. With many medical schools moving to online lectures, animated videos may complement video lectures. They can also form part of a lecture by providing a brief visual aid as part of a longer lecture, potentially helping more visual learners and keeping students' attention (Long 2014). Three animation software options are discussed below: PowToon, RawShorts and VideoScribe.

\section{PowToon}

PowToon allows users to generate short animated presentations relatively easily. Straightforward tutorials are available on the PowToon website. Once created, presentations can be uploaded to YouTube and then incorporated into a PowerPoint presentation. This requires internet access, unless the videos are downloaded in advance. PowToon operates on a 'freemium' basis. This means there is a basic package that is available for free, but additional features have to be paid for. Although monthly subscriptions are expensive, large discounts are available for teachers and students, with packages starting from US $\$ 1$ per month. PowToon is ideal for short presentations that aim to communicate one or two simple ideas (for example, 'What is the CAGE questionnaire?' or 'Helpful ideas to improve adherence'). It is less suitable for longer presentations and it would be time-consuming to use for more complex topics.

\section{RawShorts}

RawShorts is another animation software, much like PowToon, for making short animated videos. These will not replace the hour-long lecture but are ideal for a quick presentation to introduce a lecture or to put on a website. RawShorts also works on a freemium basis, although the free package is quite basic. The free version has a RawShorts watermark, and its videos are low-resolution and cannot be downloaded, only exported to YouTube. The paid-for packages can be purchased as a monthly subscription; alternatively, there is the option to pay on the basis of the number of presentations you plan to export, which is a very useful option when initially evaluating the usefulness of the software. RawShorts is a tool well worth spending some time experimenting with to see where it could be of use.

\section{VideoScribe}

VideoScribe is an impressive software tool that can be used to make visually engaging presentations. This software allows the user to create an animated sequence to go with a recorded audio lecture. It is probably best suited to short presentations but has been adapted very successfully to longer lectures and can be of great benefit to more visual learners. When done well, the engaging nature of the visual media makes the presentation very memorable. As with all of the animation softwares, the creation of these presentations may be quite time-consuming. Although a free trial is offered, there is no freemium version of VideoScribe, but it is possible to become a lifetime member for US $\$ 480$.

\section{Blackboards/whiteboards}

When looking at ways to maximise the learning experience with PowerPoint, an often overlooked option that should be mentioned is the physical blackboard/whiteboard. Presentation-based software mainly appeals to students who have visual and auditory learning styles. Students with reading/writing, interpersonal, intrapersonal or kinaesthetic learning styles may benefit from a more tutorial-based or interactive presentation (Gardner 1999). Blackboard-based teaching can be an important component of this interactive teaching. A recent study has demonstrated the effectiveness of combining PowerPoint with blackboard-based teaching for large groups (Meo 2013). Students who received integrated teaching using both PowerPoint and a blackboard had significantly better test results compared with students who received teaching with either method alone. Teaching using blackboards can be a great way of creating an interactive space during a lecture, an important component of effective learning.

\section{Alternatives to PowerPoint}

In this section, alternatives to PowerPoint are discussed. For convenience these are divided into two groups, although this is somewhat artificial. First, we discuss ICT that can be utilised to generate mind maps or flow diagrams, including a discussion on the potential educational benefits of this style of presentation. Second, we look at other slide-based software options similar to PowerPoint.

Many companies have created software that can be used to create multimedia presentations. Although unfamiliarity may be a challenge, these offer a variety of advantages, from cost to ease of the user interface. One of the potential disadvantages, which cannot be overemphasised, 
is the fact that many online sources will be blocked in certain hospitals and academic institutions. The other significant possible drawback is that some of this software will generate files that cannot be opened on certain computers. In these instances, it is important to save the document as a PowerPoint or PDF file; in fact, where possible this is always preferable. It is vital to ensure that the laptop or storage device is compatible with the computer in question. The safest way of doing this is to test it in the classroom before the lecture is delivered (Long 2014).

\section{Mind-mapping software}

Mind-mapping software, such as Prezi, allows students and teachers to create spider diagrams or visual representations of information. This presentation style may have many pedagogical advantages. The visual structuring of information may help to shape or modify a student's underlying internal knowledge structure or schema (Good 1990: p. 187). For example, seeing differential diagnoses visually displayed and grouped by their ICD-10 categories may help students to remember each of the different categories (organic, substancerelated, psychotic illness, affective illness, etc.) when formulating a differential diagnosis. A wellformed structure of knowledge is important in the application of that knowledge to problem-solving (Kaufman 2013).

Although learning style theory has its critics (Coffield 2004), individual students each have their different style of learning; over-relying on one type may disadvantage students who use different learning styles (Stitt-Gohdes 2001). Mind-mapping technology can be very useful when trying to cater to both convergent and divergent thinkers (Hudson 1966). Convergent thinkers tend to follow a linear, logical argument to a single correct answer. Divergent thinkers, on the other hand, think in many varied directions; their thought process is more flexible. It is important to cater to both styles of learning, as there is some evidence that both convergent and divergent learners learn better from teachers who use the style that the students use themselves (Joyce 1968). The linear nature of PowerPoint tends to appeal more to the convergent mind. Mind maps may allow students to approach learning from the perspective they find easier to use. They can start with a single symptom and work towards a range of different diagnoses. For example, presented with the symptom of bizarre behaviour, the student can form the differential diagnosis of mania, psychosis, drug intoxication, etc. Alternatively, students can start with a range of signs and symptoms and work towards a single diagnosis. This can all be depicted visually using mind-mapping technology.

Pask (1976) suggested two similar learning styles: serialistic and holistic. Serialists - similar to convergent learners - grow their schema from basic concepts and simple facts, and holists develop an overall framework. Mind-mapping technology can appeal to both learning styles, whereas PowerPoint presentations more closely fit the serialist's learning style.

\section{Prezi}

Prezi's presentation software was launched in 2009 and now has over 50 million users. It allows presenters to create a mind map or spider diagram on a large canvas like a blackboard; each area of this canvas can be zoomed in on in turn. Prezi was first mentioned in the medical literature in 2012 (Tam 2012). A brief study looking at students' experiences of using Prezi has highlighted some of it strengths and weaknesses (Duffy 2014). The main benefits of Prezi are its useful overview and its ability to engage students. The main drawbacks are technical problems resulting from students' unfamiliarity with the software. To date, the objective benefits of using Prezi have not been investigated.

A large potential benefit of this presentation style is that the relationship between different topics can be depicted visually by their proximity to each other and by arrows connecting them. This can help to build and refine cognitive schema (Good 1990: p. 187). The relative importance of different topics can also be depicted by the physical size of the information on the Prezi canvas. This second point is especially useful in the light of cognitive load theory (Sweller 1994; van Merriënboer 2010). This theory states that working memory, or short-term memory, relies on the content of our long-term memory. Presenting too much new information that is not related to information currently in long-term memory can inhibit learning. The visual depiction of overviews of topics and knowledge can allow a student to learn the overall structure without becoming overloaded by the finer details. The relative importance of each slide can be represented by its size or position on the overall canvas. This may help students to give more likely differential diagnoses before rarer but more memorable ones. Students can also return to the lecture later and review the more complex additional information.

Prezi works on a freemium basis, requiring a monthly subscription for advanced features. Unlike many other presentation tools, the basic free package is very useful and allows the creation 
B0X 2 Good practice when using Prezi

\section{Creating a Prezi}

- Make use of online tutorials available on the Prezi website

- Carefully plan the visual layout and make it logical - use size and position to demonstrate the relative importance and 'connectedness' of different ideas

- Avoid prioritising form over content

- Keep movement to a minimum to avoid distraction and confusion

- Use multimedia clips - these may require internet access if they are being streamed from the internet

- Save local copies of presentations in the event of problems with the internet connection

- Check the privacy settings to prevent sensitive information from being freely accessed

Prezi for student use

- Spend time introducing students to the software and make IT support available to them

- Provide students with a printable version of lectures

- Advise students to create a Prezi account, as this will allow them to download and edit presentations

(Adapted from Duffy et al, 2014)

of excellent presentations. When using the most basic package, all presentations are visible to the public. Individuals involved in education and students can subscribe to receive a basic education package for free; this allows private storage. It is easy to use, and video tutorials are available on the Prezi website. Existing PowerPoint presentations can be easily imported into Prezi; however, lectures cannot be exported as PowerPoint slides, although they can be exported as PDF slides for handouts. Useful tips for creating a Prezi presentation are included in Box 2.

\section{Some other softwares}

Other free mind-mapping tools include Popplet, FreeMind and Coggle. These are excellent tools for creating flow charts and decision trees, and for depicting the structure of an organisation or the relationship between different topics. Two of the better (but not free) tools on the market are Mindjet and XMind.

\section{Slide-based presentation software}

\section{Apache OpenOffice}

OpenOffice is is free, downloadable software. It is run by the the Apache Software Foundation, a volunteer-based foundation established in 1999.
As the software can be installed on a computer, documents can be created and edited offline. OpenOffice is highly compatible with PowerPoint: files can be both imported and exported as PowerPoint files. This is a great option for people who prefer open-source software.

\section{Google Slides}

Google Slides is free cloud-based software. It is straightforward to use and does not require downloading any software onto a computer, as the user works on the presentation online. Files created using Google Slides can be exported both as PDF files or PowerPoint presentations. It is also possible to store data online, which is advantageous for sharing lecture content with others but could cause problems in relation to data protection. Therefore it may not be ideal for case presentations or presentations that include patient information.

\section{Haiku Deck}

Haiku Deck is a great piece of software. It is useful for making quick, simple, visually impressive presentations in which the presenter will be verbally conveying the majority of the information, rather than for creating text-heavy slides. This software is free, and slides can be stored privately in a cloud without having to pay a monthly subscription. There is no limit to the storage capacity. It is worth noting that even if files are stored privately in cloud storage, local data protection agencies may need to be contacted if patient information is included in slides. One of the main drawbacks is that there is no straightforward way to convert PowerPoint files into a Haiku Deck. It is possible, but it is very time-consuming. Presentations can be exported as PowerPoint slides or saved as PDF files.

\section{Keynote}

Keynote is a useful alternative for Mac users. Its templates are aesthetically superior to those in PowerPoint. Some advantages of this software have been described in radiology, where the use of high-resolution images from different sources is necessary (Ghoshhajra 2011). It is worth noting that it is possible to convert Keynote presentations into PowerPoint, but it can be problematic for all but the simplest of presentations. There are some subtle differences between Keynote and PowerPoint: the latter is superior at handling vector graphics, which means that images may appear clearer and higher-resolution images may take up less storage space. On the other hand, Keynote has better typography. 


\section{MCQ answers}

$1 \mathrm{c} \quad 2$ e 3 e $\quad 4$ b $5 d$
Connecting an Apple computer to a projector is not always a straightforward task. Most Apple laptops currently in use have a Mini DisplayPort that connects to the VGA port on a projector consequently, a Mini DisplayPort-VGA adaptor will be needed. Occasionally, there will be a need for an adaptor connecting a Mini DisplayPort to an HDMI or DVI port; this is more likely where a flat-screen television is being used in place of a projector. The safest course of action is to test your laptop in the appropriate location well in advance of the presentation.

\section{Presentation Free 2013}

Presentation Free 2013 is free software that can be downloaded onto your computer. It is possible to edit and store files offline, so this software is appropriate for presentations that contain patient information, and it is not dependent on internet access. It is fully compatible with PowerPoint.

\section{Conclusions}

PowerPoint can be a useful tool in supporting a well-planned lecture. However, we should not limit ourselves to PowerPoint. There are alternatives that may be more appropriate, and there are also tools that when used in conjunction with PowerPoint enhance and broaden the learning experience. There are alternatives to PowerPoint with a range of strengths and weaknesses. Individuals considering using alternative technologies should be mindful of the issues of cloud-based storage and freemium payment options, and consider strategies for supporting students in using new technologies. Software that facilitates the generation of mind maps may confer pedagogical advantages in certain contexts and may suit a wider range of learning styles. Animation-based software may be a good addition to a PowerPoint presentation or may be used to replace shorter presentations. Interactive technology is an exciting emerging area and has the potential to provide teachers with real-time data on their students' understanding, and to provide learners with instantaneous feedback. Technology, however, is no substitute for a well-prepared, passionate lecturer who cares deeply about the topic and the students.

\section{References}

Beebe CR, Gurenlian JR, Rogo EJ (2014) Educational technology for millennial dental hygiene students: a survey of U.S. dental hygiene programs. Journal of Dental Education, 78: 838-49.

Bligh D (2000) What's the Use of Lectures? Jossey-Bass.

Buckingham, D (2007) Beyond Technology: Children's Learning in the Age of Digital Culture. Polity.

Cantillon P (2003) Large group teaching. BMJ, 326: 437.
Clark RC, Mayer RE (2011) e-Learning and the Science of Instruction: Proven Guidelines for Consumers and Designers of Multimedia Learning. Wiley.

Coffield F, Moseley D, Hall E, Ecclestone K (2004) Learning Styles and Pedagogy in Post-16 Learning. A Systematic and Critical Review. Learning and Skills Research Centre.

Craig RJ, Amernic JH (2006) PowerPoint presentation technology and the dynamics of teaching. Innovative Higher Education, 31: 147-60.

Duffy RM, Gurandel A, Casey P, et al (2014) Experiences of using Prezi in psychiatry teaching. Academic Psychiatry, 39: 615-9.

Duncan D (2005) Clickers in the Classroom: How to Enhance Science Teaching Using Classroom Response Systems. Pearson Education.

Dunkin MJ (1983) A review of research on lecturing. Higher Education Research and Development, 2: 63-78.

Garber AR (2001) Death by Powerpoint. Small Business Computing (http://www.smallbusinesscomputing.com/biztools/article. php/684871/Death-By-Powerpoint.htm). Accessed 23 September 2016

Gardner H (1999) Intelligence Reframed. Multiple Intelligences for the 21st Century. Basic Books.

Ghoshhajra BB, Techasith T, Choy G, et al (2011) Using Keynote to present radiology images. Journal of Digital Imaging, 24: 844-7.

Good TL, Brophy JE (1990) Educational Psychology: A Realistic Approach (4th edn). Longman.

Henry M (2015) Learning in the digital age: developing critical, creative and collaborative skills. In Teaching and Learning with ICT in the Primary School (eds S Younie, M Leask, K Burden): pp. 1-12. Routledge.

Hilty DM, Alverson DC, Alpert JE, et al (2006) Virtual reality, telemedicine, web and data processing innovations in medical and psychiatric education and clinical care. Academic Psychiatry, 30: 528-33.

Hudson L (1966) Contrary Imaginations. Penguin.

Joyce C, Hudson L (1968) Student styles and teacher styles: an experimental study. British Journal of Medical Education, 2: 28-32.

Kaufman DM, Mann KV (2013) Teaching and learning in medical education: how theory can inform practice. In Understanding Medical Education: Evidence, Theory and Practice (2nd edn) (ed T Swanwick): pp. 7-32. Blackwell Publishing.

Long A, Lock B (2014) Lectures and large groups. In Understanding Medical Education: Evidence, Theory and Practice (2nd edn) (ed T Swanwick): p. 139-50. Blackwell Publishing.

Meo SA, Shahabuddin S, Al Masri AA, et al (2013) Comparison of the impact of PowerPoint and chalkboard in undergraduate medical teaching: an evidence based study. Journal of the College of Physicians and Surgeons Pakistan, 23: 47-50.

Michel JB, Shen YK, Aiden AP, et al (2011) Quantitative analysis of culture using millions of digitized books. Science, 331: 176-82.

Pask G (1976) Styles and strategies of learning. British Journal of Educational Psychology, 46: 128-48.

Revell SM, McCurry MK (2010) Engaging millennial learners: effectiveness of personal response system technology with nursing students in small and large classrooms. Journal of Nursing Education, 49: $272-5$.

Roopa S, Geetha MB, Rani A, et al (2013) What type of lectures students want? A reaction evaluation of dental students. Journal of Clinical and Diagnostic Research, 7: 2244-6.

Selwyn N (2011) Education and Technology: Key Issues and Debates. Continuum.

Stitt-Gohdes W (2001) Business education students' preferred learning styles. Journal of Career and Technical Education, 18: 32-45.

Stokes K (2012) Decoding learning: the proof, promise and potential of digital education. Education Journal, 149: 8-12. 
Sweller J (1994) Cognitive load theory, learning difficulty, and instructional design. Learning and Instruction, 4: 295-312.

Tam CWM, Eastwood A (2012) Available, intuitive and free! Building e-learning modules using web 2.0 services. Medical Teacher, 34: 1078-80.

Undrill G, McMaster F (2013) PowerPoint: avoiding the slide to damnation. Advances in Psychiatric Treatment, 19: 14-22. van Merriënboer JJ, Sweller J (2010) Cognitive load theory in health professional education: design principles and strategies. Medical Education, 44: 85-93.

Wessels A, Fries S, Horz H, et al (2007) Interactive lectures: effective teaching and learning in lectures using wireless networks. Computers in Human Behavior, 23: 2524-37.

\section{MCQs}

Select the single best option for each question stem

1 The alternatives to PowerPoint:

a are safer to use for presentations including sensitive information

b are much easier for students to use

c are often free or have additional features that PowerPoint does not have

d offer freemium subscriptions, which means you only pay for the services that you need

e use cloud-based file storage, so you do not need internet access to edit your presentation.

\section{When using Prezi:}

a extensive use of movement helps to engage learners

b students should not need advice on how to use the software, as modern students are highly computer literate and Prezi is very intuitive c internet access is never needed to present a lecture

$\mathrm{d}$ educational packages are not available for students and teachers

e consideration should be given to the suitability of Prezi if there is patient information in a presentation, as presentations are stored online.

\section{Animation software:}

a is a good option for long, complex topics

b greatly reduces the time required to prepare a presentation

c is not well developed enough to make it easily accessible to the user

d includes many high-quality packages that are available free of charge

e can be useful for creating an introduction to a topic.

\section{Personal response systems:}

a do not give the lecturer real-time information on students' understanding

b are able to provide the student with real-time feedback

c encourage passive learning

d are easy to set up

e are free to download.

5 Mind-mapping technology:

$\mathrm{a}$ is expensive and complicated to use

$\mathrm{b}$ is only of benefit when the learner has a wellformed structure of knowledge

c is more likely to result in cognitive overload compared with standard presentations

d can help to develop cognitive schema

e disadvantages visual learners. 